\title{
Synchronous Peripancreatic Lymph Node Gastrinoma and Gastric Neuroendocrine Tumor Type 2
}

\author{
Hee Woo Lee', Jun-Won Chung', Yoon Jae Kim¹, Kwang Ahn Kwon', Eui Joo Kim¹, Keon Kuk Kim², Woon Ki Lee ${ }^{2}$ and Sun Jin Sym³ \\ Departments of ${ }^{1}$ Internal Medicine, ${ }^{2}$ Surgery, and ${ }^{3}$ Hematology, Gachon University Gil Medical Center, Gachon University College of \\ Medicine, Incheon, Korea
}

\begin{abstract}
A 34-year-old man was referred to our hospital with gastric polypoid lesions and biopsy-confirmed neuroendocrine tumor (NET). Computed tomography $(\mathrm{CT})$ revealed a $3 \times 3.5 \times 8-\mathrm{cm}$ retroperitoneal mass behind the pancreas, with multiple hepatic metastases. His serum gastrin level was elevated to $1,396 \mathrm{pg} / \mathrm{mL}$. We performed a wedge resection of the stomach, a right hemi-hepatectomy, and a retroperitoneal mass excision. After careful review of the clinical, radiological, histopathological, and immunohistochemical findings, peripancreatic gastrinoma, and synchronous gastric NET were ultimately diagnosed. We reviewed a CT scan that had been performed 6 years previously after surgery for a duodenal perforation. There was no evidence of gastric or hepatic lesions, but the retroperitoneal mass was present at the same site. Had gastrinoma been detected earlier, our patient could have been cured using less invasive treatment. This case demonstrates how important it is to consider Zollinger-Ellison syndrome in patients with a recurrent or aggressive ulcer. Clin Endosc 2016;49:483-487
\end{abstract}

Key Words: Gastrinoma; Neuroendocrine tumors; Zollinger-Ellison syndrome

\section{INTRODUCTION}

Gastrinomas that cause Zollinger-Ellison syndrome (ZES) occur commonly in the gastrinoma triangle, whose vertices lie at the junction of the cystic and common bile ducts, the junction of the second and third parts of the duodenum, and the junction of the neck and body of the pancreas. ${ }^{1}$ Many case studies have suggested that lymph nodes can be the primary site of gastrinoma; nonetheless, this proposition remains controversial. $^{2-5}$

In patients who have a gastric neuroendocrine tumor (NET), gastrinoma should always be considered and ruled out, because type 2 gastric NETs are often caused by gastrin-secreting

Received: December 15, 2015 Revised: March 7, 2016

Accepted: April 25, 2016

Correspondence: Jun-Won Chung

Division of Gastroenterology, Department of Internal Medicine, Gachon University Gil Medical Center, Gachon University College of Medicine, 21 Namdong-daero 774beon-gil, Namdong-gu, Incheon 21565, Korea

Tel: +82-32-460-2791, Fax: +82-32-460-3408, E-mail: junwonchung@hanmail.net

(cc) This is an Open Access article distributed under the terms of the Creative Commons Attribution Non-Commercial License (http://creativecommons.org/ licenses/by-nc/3.0) which permits unrestricted non-commercial use, distribution, and reproduction in any medium, provided the original work is properly cited. neoplastic tissue of the kind that occurs in ZES, which in turn is commonly the result of a duodenal or pancreatic gastrinoma. ${ }^{6-8}$ Herein, we report a case of rare peripancreatic lymph node gastrinoma that was found during a workup of gastric NET.

\section{CASE REPORT}

A 34-year-old man was referred to our hospital with a gastric polypoid lesion and biopsy-confirmed NET. He had undergone surgery for a duodenal perforation 6 years prior to the diagnosis of NET, but he had been lost to follow-up. He complained of epigastric pain and dyspepsia. There were no abnormal findings upon physical examination, and the results of peripheral blood tests, routine chemistry, and tumor marker tests were within normal limits. The serum gastrin level was elevated to $1,396 \mathrm{pg} / \mathrm{mL}$ (reference range, 0 to 100). However, a thyroid function test and a low-dose overnight dexamethasone suppression test were normal, as were as levels of parathyroid hormones, calcitonin, glucagon, growth hormone, and insulin-like growth factor. 

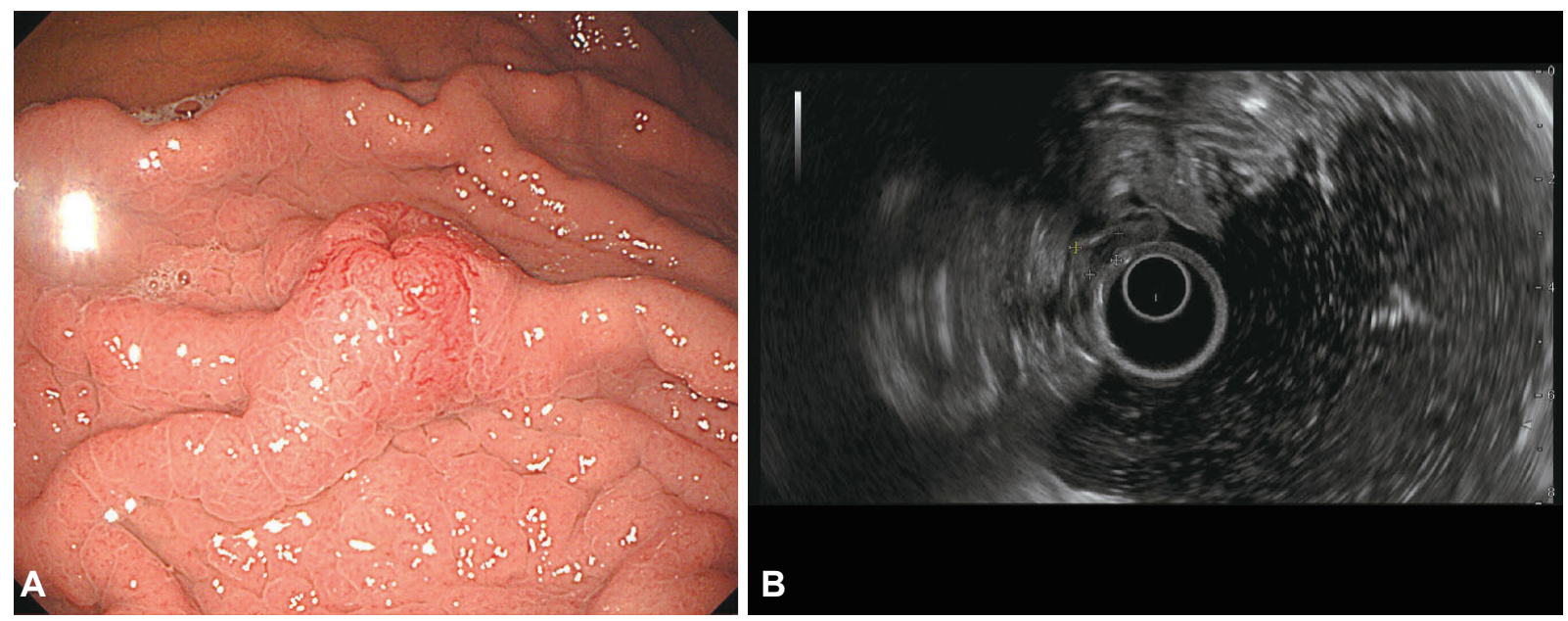

Fig. 1. Endoscopic imaging. (A) Esophagogastroduodenoscopy showing a localized hyperemic elevated lesion, with central umbilication, located at the greater curvature of the gastric high body. (B) Endoscopic ultrasonography revealing an $8 \times 9-\mathrm{mm}$, oval shaped, homogeneous, hypoechoic lesion that originated from the submucosal layer.
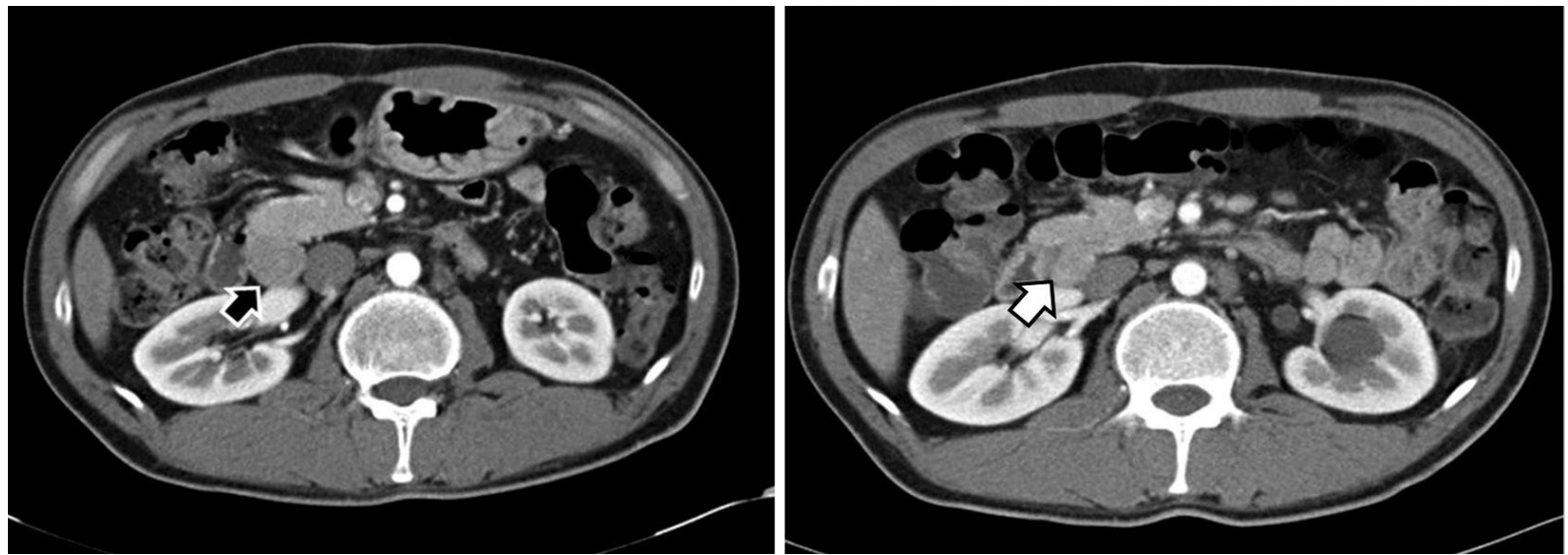

Fig. 2. Computed tomography (CT). (A) CT revealing a $3 \times 3.5 \times 8-\mathrm{cm}$ homogeneous retroperitoneal mass behind the pancreas (black arrow). (B) CT performed 6 years prior to diagnosis showing that the retroperitoneal mass was present at the same site, and had been overlooked (white arrow).

An esophagogastroduodenoscopy revealed a localized hyperemic elevated lesion, with central umbilication, located at the greater curvature of the gastric high body (Fig. 1A). An endoscopic ultrasonography revealed an $8 \times 9-\mathrm{mm}$, ovular, homogeneous, hypoechoic lesion that originated from the submucosal layer (Fig. 1B).

Computed tomography (CT) of the abdomen revealed multiple small, peripheral, enhanced, hypodense lesions in S6 of the liver, indicative of hepatic metastasis. In addition, we found a $3 \times 3.5 \times 8-\mathrm{cm}$ homogeneous retroperitoneal mass, with smooth boundaries and without contrast enhancement. The lesion was located behind the pancreas and was dissociated from pancreatic and liver tissue (Fig. 2A). Liver magnetic resonance imaging revealed several additional liver lesions with slightly low signal intensity on T1-weighted images, and high signal intensity on T2-weighted images (Fig. 3). A biopsy of these hepatic lesions confirmed NET grade 2 .
We reviewed a CT scan that had been performed 6 years previously after surgery for a duodenal perforation. There was no evidence of the gastric or hepatic lesions, but the retroperitoneal mass was present at the same site, and had been overlooked (Fig. 2B).

After careful review of the clinical and radiological findings, we diagnosed peripancreatic gastrinoma and synchronous gastric NET in this patient.

We performed a wedge resection of the stomach, a right hemi-hepatectomy with cholecystectomy, and a retroperitoneal mass excision. Histological examination revealed that the removed gastric mass was NET grade 2, and that it was composed of uniform cells with round or ovoid nuclei and scant eosinophilic cytoplasm; the cells were proliferating in a trabecular or glandular pattern. The tumor cells had invaded the submucosal layer, and they stained diffusely for chromogranin A and synaptophysin. The mitotic count was 3 per 

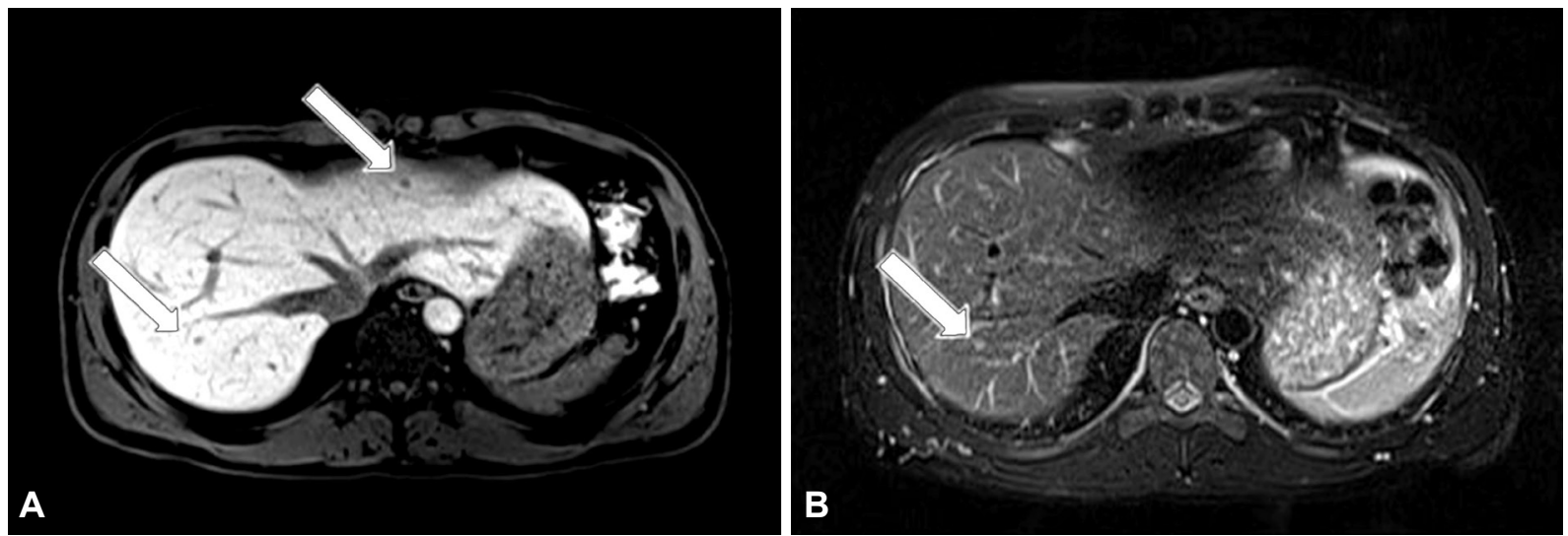

Fig. 3. Liver magnetic resonance imaging. Liver magnetic resonance imaging demonstrating (arrows) several additional liver lesions with slightly (A) low signal intensity on T1-weighted images, and (B) high signal intensity on T2-weighted images.
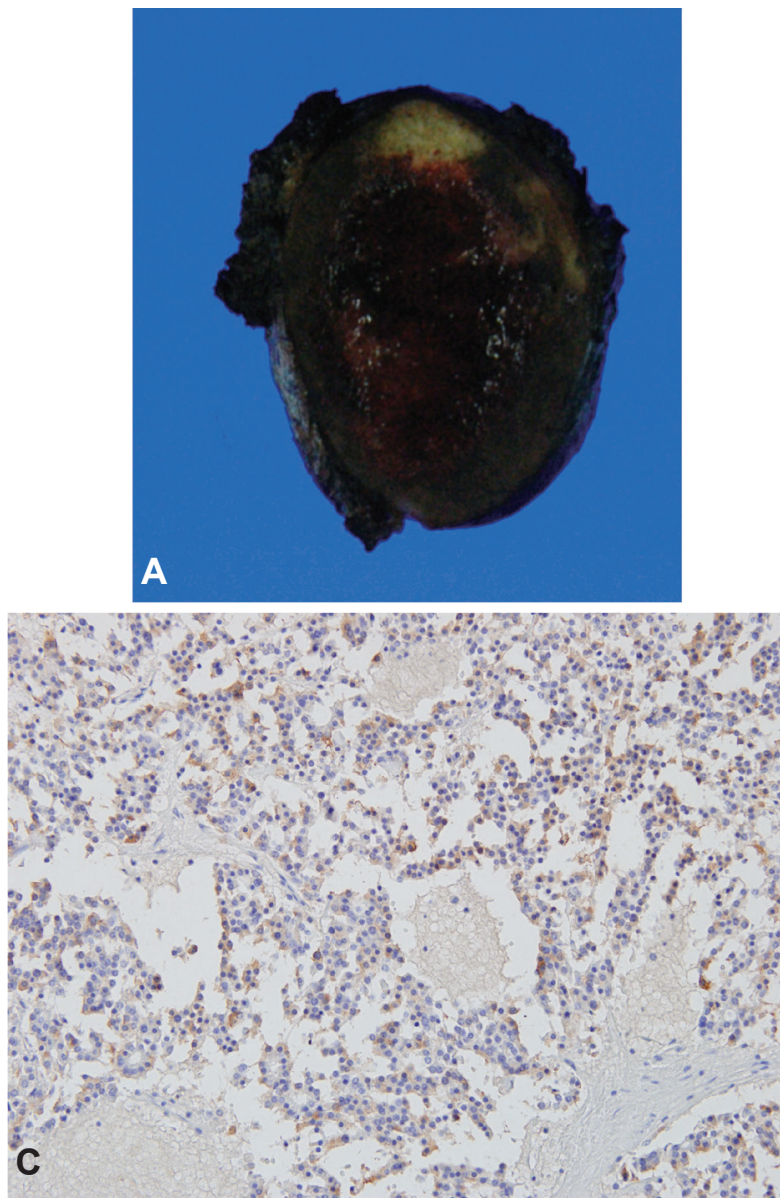
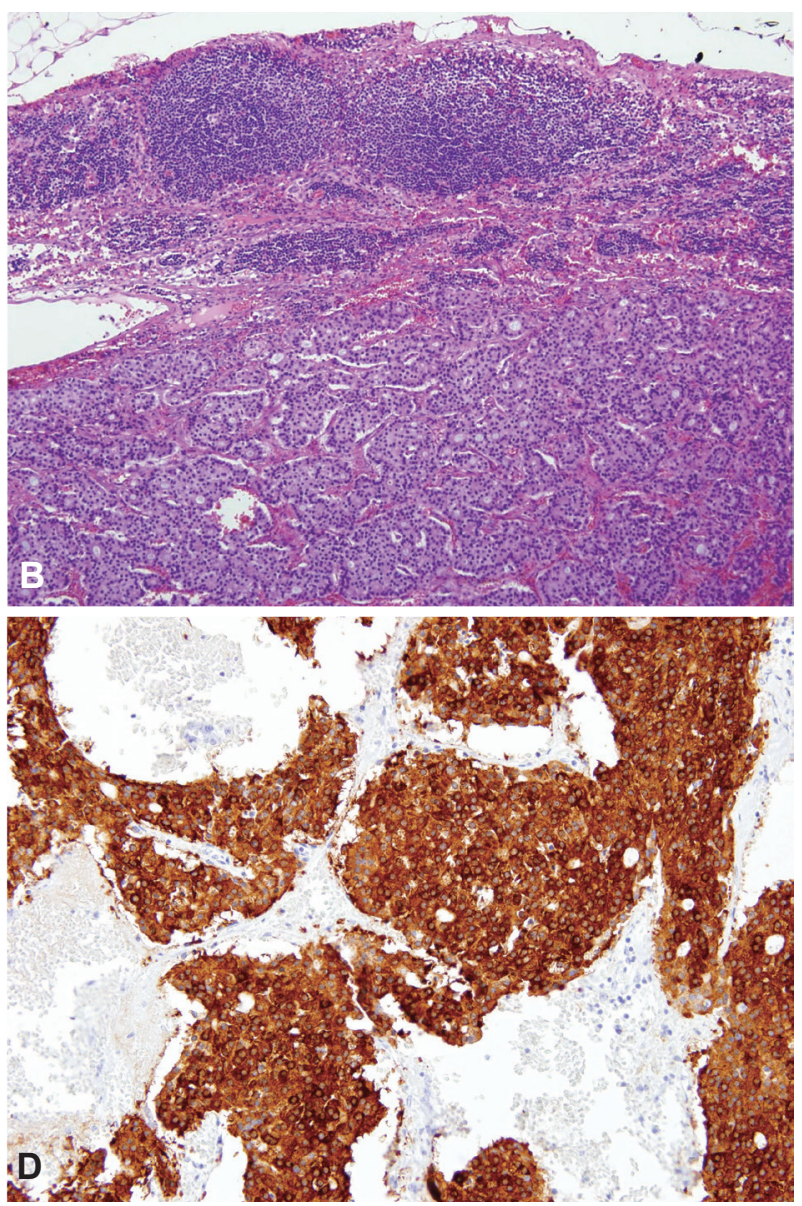

Fig. 4. Histologic examinations of retroperitoneal lymph nodes. (A) The resected lymph node showing a well-demarcated, solid grayish-tan lesion, $8 \times 3 \mathrm{~cm}$ in size, with punctuate foci of hemorrhage. $(B) H \& E$ stain $(\times 100)$ demonstrating that the tumor was composed of an organoid nest, and that it had trabecular growth pattern. Immunohistochemically, the tumor cells were reactive for $(C)$ chromogranin $A(\times 200)$ and $(D)$ synaptophysin $(\times 200)$.

10 high-power fields (HPF), and the Ki-67 index was 1\%. Both the lateral and vertical resection margins were free from tumor involvement.

The removed hepatic mass was diagnosed as NET grade 2 upon histological examination. It stained positively for chromogranin A and synaptophysin. The mitotic count was $3 / 10$ HPF, and the Ki-67 index was $1 \%$.

Gross examination of the retroperitoneal mass showed a 
well-demarcated, solid, grayish-tan lesion of $3 \times 3.5 \times 8 \mathrm{~cm}$, with punctuate foci of hemorrhage. Microscopically, the tumor was composed of an organoid nest and had a trabecular growth pattern. The tumor cells had round and vesicular nuclei, with finely granular and dusty chromatin, and multiple nucleoli. Immunohistochemically, the tumor cells were reactive for chromogranin A and synaptophysin (Fig. 4). The mitotic count was 3/10 HPF, and the Ki-67 index was $1 \%$. The histopathological and immunohistochemical findings indicated NET grade 2. The gastrin level normalized after the operation; the patient was prescribed octreotide, and was treated on an outpatient basis.

Six months after surgery, the patient's fasting gastrin level was within normal limits, and a provocative secretin-stimulation test was normal.

\section{DISCUSSION}

Herein, we have reported a case of rare synchronous peripancreatic lymph node gastrinoma combined with type 2 gastric NET. Type 2 gastric NET often occurs in patients with ZES or multiple endocrine neoplasia type 1 (MEN-1), and it is primarily caused by a duodenal gastrinoma. Although we performed laboratory tests to screen for MEN-1, there were no abnormal findings. Furthermore, we found none of the following symptoms: weight loss, headache, nausea, constipation, diarrhea, hypercalcemia, hypoglycemia, and anemia.

Gastrinoma is sometimes found in lymph nodes without a known primary tumor. Although it is not certain whether the neoplasm is primary or metastatic, many reports have suggested that primary lymph node gastrinoma does exist. ${ }^{2-5}$ For instance, Jang et al. ${ }^{9}$ reported one case of primary peripancreatic lymph node gastrinoma in South Korea.

When a gastrinoma is found in a lymph node, it is often difficult to diagnose whether the tumor is a primary or metastatic lesion. Some studies have suggested that the main diagnostic criteria of primary lymph node gastrinoma are: normalization of the gastrin level after resection of lymph node gastrinoma, no evidence of another primary tumor, continuous normal gastrin levels, and lack of any symptoms. ${ }^{10,11}$ In our case, the patient's gastrin level decreased after excision of the peripancreatic lymph node mass and hepatic masses.

The main dilemma in our case was whether the peripancreatic lymph node was primary. Nonetheless, we considered the peripancreatic lymph node to be the primary site, because the small gastric NET was limited to the submucosal layer, and the multiple hepatic lesions were considered metastases. In addition, a peripancreatic mass was present on the previous CT scan, while the stomach and liver were clear.
There are limitations to our case; first, we did not meticulously explore the duodenum, nor did we carry out a regional lymphadenectomy at the time of surgery. Second, we did not perform an octreotide scan to search for another primary tumor. Third, the relatively short-term (6-month) follow-up period (rather than 1 year) was not enough to rule out occult primary disease, which requires long-term follow-up.

Hepatic metastases are the most important predictor of survival in patients with ZES and other gastrointestinal NETS. ${ }^{12-}$

${ }^{14}$ It is important to detect gastrinoma early, because the size of the primary tumor is an important factor when predicting liver metastases. ${ }^{12-15}$ More specifically, if gastrinoma had been detected earlier in the present case, our patient could have been cured using a less-invasive treatment.

Surgery is an important management option in advanced gastrinoma with NET, both for survival and symptom control, even if curative resection is not possible. In various studies, a 5 -year survival rate of $71 \%$ to $85 \%$ has been reported, with aggressive surgical resection of liver metastases, compared to $20 \%$ to $30 \%$ if left untreated. ${ }^{16-20}$ Therefore, liver resection in advanced gastrinoma can be performed with acceptable morbidity and low mortality rates.

In summary, we have described the case of a 34-year-old man with synchronous peripancreatic lymph node gastrinoma and type 2 gastric NET. This case demonstrates the importance of considering ZES in patients with a recurrent or aggressive ulcer.

Conflicts of Interest

The authors have no financial conflicts of interest.

\section{REFERENCES}

1. Passaro E Jr, Howard TJ, Sawicki MP, Watt PC, Stabile BE. The origin of sporadic gastrinomas within the gastrinoma triangle: a theory. Arch Surg 1998;133:13-16.

2. Mian O, Mahmoud A, Ibrahim M, Rassai H. Primary gastrinoma of lymph node: fact or fiction? Am Surg 2009;75:208-211.

3. Norton JA, Alexander HR, Fraker DL, Venzon DJ, Gibril F, Jensen RT. Possible primary lymph node gastrinoma: occurrence, natural history, and predictive factors: a prospective study. Ann Surg 2003;237:650-657.

4. Anlauf M, Enosawa T, Henopp T, et al. Primary lymph node gastrinoma or occult duodenal microgastrinoma with lymph node metastases in a MEN1 patient: the need for a systematic search for the primary tumor. Am J Surg Pathol 2008;32:1101-1105.

5. Herrmann ME, Ciesla MC, Chejfec G, DeJong SA, Yong SL. Primary nodal gastrinomas. Arch Pathol Lab Med 2000;124:832-835.

6. Crosby DA, Donohoe CL, Fitzgerald L, et al. Gastric neuroendocrine tumours. Dig Surg 2012;29:331-348.

7. Berna MJ, Annibale B, Marignani M, et al. A prospective study of gastric carcinoids and enterochromaffin-like cell changes in multiple endocrine neoplasia type 1 and Zollinger-Ellison syndrome: identification of risk factors. J Clin Endocrinol Metab 2008;93:1582-1591.

8. Norton JA, Melcher ML, Gibril F, Jensen RT. Gastric carcinoid tumors in multiple endocrine neoplasia-1 patients with Zollinger-Ellison syn- 
drome can be symptomatic, demonstrate aggressive growth, and require surgical treatment. Surgery 2004;136:1267-1274.

9. Jang SH, Jang SM, Jun YJ, et al. Peripancreatic lymph node gastrinoma. Basic Appl Pathol 2009;2:140-142.

10. Howard TJ, Zinner MJ, Stabile BE, Passaro E Jr. Gastrinoma excision for cure. A prospective analysis. Ann Surg 1990;211:9-14

11. Zhou H, Schweikert HU, Wolff M, Fischer HP. Primary peripancreatic lymph node gastrinoma in a woman with MEN1. J Hepatobiliary Pancreat Surg 2006;13:477-481.

12. Strosberg J, Gardner N, Kvols L. Survival and prognostic factor analysis of 146 metastatic neuroendocrine tumors of the mid-gut. Neuroendocrinology 2009;89:471-476.

13. Yu F, Venzon DJ, Serrano J, et al. Prospective study of the clinical course, prognostic factors, causes of death, and survival in patients with long-standing Zollinger-Ellison syndrome. J Clin Oncol 1999;17:615630 .

14. Cadiot G, Vuagnat A, Doukhan I, et al. Prognostic factors in patients with Zollinger-Ellison syndrome and multiple endocrine neoplasia type 1. Groupe d'Etude des Neoplasies Endocriniennes Multiples (GENEM and groupe de Recherche et d'Etude du Syndrome de Zollinger-Ellison (GRESZE). Gastroenterology 1999;116:286-293.

15. Jensen RT, Niederle B, Mitry E, et al. Gastrinoma (duodenal and pancreatic). Neuroendocrinology 2006;84:173-182.

16. Bartlett EK, Roses RE, Gupta M, et al. Surgery for metastatic neuroendocrine tumors with occult primaries. J Surg Res 2013;184:221-227.

17. Capurso G, Rinzivillo M, Bettini R, Boninsegna L, Delle Fave G, Falconi M. Systematic review of resection of primary midgut carcinoid tumour in patients with unresectable liver metastases. Br J Surg 2012;99:14801486.

18. Givi B, Pommier SJ, Thompson AK, Diggs BS, Pommier RF. Operative resection of primary carcinoid neoplasms in patients with liver metastases yields significantly better survival. Surgery 2006;140:891-897.

19. Touzios JG, Kiely JM, Pitt SC, et al. Neuroendocrine hepatic metastases: does aggressive management improve survival? Ann Surg 2005;241:776783.

20. Norton JA, Kivlen M, Li M, Schneider D, Chuter T, Jensen RT. Morbidity and mortality of aggressive resection in patients with advanced neuroendocrine tumors. Arch Surg 2003;138:859-866. 